\title{
Magneto- and Electroresistance of Ultrathin Anisotropically Strained La-Sr-MnO Films
}

\author{
S. BAlevičius ${ }^{a}$, V. Stankevič ${ }^{a}$, N. Žurauskiene $\dot{\mathrm{Z}}^{a, *}$, \\ Č. ŠimkeviČIUs ${ }^{a}$, J. PARŠEliŪnas ${ }^{a}$, P. Cimmperman ${ }^{a}$, \\ A. Abrutis ${ }^{b}$ And V. Plaušinaitiene $\dot{E}^{b}$ \\ ${ }^{a}$ Semiconductor Physics Institute, A. Goštauto 11, Vilnius, Lithuania \\ ${ }^{b}$ Vilnius University, Dept. of General and Inorganic Chemistry \\ Naugarduko 24, Vilnius, Lithuania
}

\begin{abstract}
The magnetoresistance anisotropy of ultrathin $\mathrm{La}_{0.83} \mathrm{Sr}_{0.17} \mathrm{MnO}_{3}$ films deposited on $\mathrm{NdGaO}_{3}$ substrate by metalorganic chemical vapour deposition technique was investigated. The electric-field-induced resistance change was studied up to electric fields of $10 \mathrm{kV} / \mathrm{cm}$ using ns duration electrical pulses. It was found that in ultrathin $(<10 \mathrm{~nm})$ and thin $(>50 \mathrm{~nm})$ films the origin of electric-field-induced resistance change is thermal. However, the films with thicknesses of about $20 \mathrm{~nm}$, exhibit negative electric-field-induced resistance change, having a pure electronic nature. This effect is explained in terms of two-layer systems with imperfections located at the interface between the layers.
\end{abstract}

PACS numbers: 75.47.Gk, 75.30.Gw, 73.50.-h, 68.55.Jk

\section{Introduction}

The high Curie temperature of $\mathrm{La}-\mathrm{Sr}-\mathrm{MnO}_{3}$ makes it an attractive material for vertical tunnel or artificial planar junction devices $[1,2]$. However, further development of these devices requires better knowledge of the behaviour of the material at each interface, i.e. substrate/film, vertical junctions, or artificial structure interfaces [2]. This can be accomplished by studying electrical transport in $\mathrm{La}-\mathrm{Sr}-\mathrm{MnO}_{3}$ ultrathin films. Such investigations revealed a strong increase in electrical resistivity of these films when film thickness was decreased below $20 \mathrm{~nm}$ [3].

*corresponding author; e-mail: zurausk@pfi.lt 
This $20 \mathrm{~nm}$ thick transition layer, called the "dead layer" [2], is responsible for thickness-dependent resistivity changes in ultrathin films.

In this paper, properties of the "dead layer" of moderate thickness (few tens of $\mathrm{nm}$ ) and its influence on the transport properties of $\mathrm{La}_{0.83} \mathrm{Sr}_{0.17} \mathrm{MnO}_{3}$ films deposited on $\mathrm{NdGaO}_{3}$ (NGO) substrates by metalorganic chemical vapour deposition (MOCVD) technique are presented.

\section{Experiment}

The $\mathrm{La}_{0.83} \mathrm{Sr}_{0.17} \mathrm{MnO}_{3}$ films were prepared on $\mathrm{NdGaO}_{3}$ (001) substrates at $825^{\circ} \mathrm{C}$ using MOCVD from a liquid source solution having the composition $\mathrm{La}_{0.78} \mathrm{Sr}_{0.22} \mathrm{Mn}_{0.733}$. The thickness of the films ranged from 4 to $60 \mathrm{~nm}$. $6-8 \mathrm{~mm}$ long and 2-5 mm wide samples were co-planar with thin Ag electrodes placed at $d=50 \mu \mathrm{m}$ distance relative to each other. These electrodes were deposited by thermal evaporation at $200^{\circ} \mathrm{C}$ and then annealed in an argon gas atmosphere at $400^{\circ} \mathrm{C}$. Low-field resistance and magnetoresistance (MR) were measured at temperatures ranging from 5 to $300 \mathrm{~K}$ by applying dc electric and magnetic fields with a maximum of the latter of $0.8 \mathrm{~T}$. To measure the electric-field-induced resistance change ("electroresistance" ER), the samples were connected in series to a $50 \Omega$ impedance $18 \mathrm{GHz}$ frequency transmission line and pulsed by $5-10 \mathrm{~ns}$ ( $0.5 \mathrm{~ns}$ rise time) rectangularly shaped electrical pulses with amplitudes up to $500 \mathrm{~V}$.

The film structure was investigated using reflection high-energy electron diffraction (RHEED).

\section{Results and discussion}

The RHEED patterns recorded along the [110] zone-axis (with regard to the substrate) of a $4 \mathrm{~nm}$ sample are shown in Fig. 1a. Spots from the substrate have elongated shapes, in contrast to spots from the film, which have more rounded shapes with a little elongation in a direction parallel to the surface. Such shapes correspond to an orthorhombic face-centred phase structure with a lattice constant of $a=4.06 \AA$ in plane and $c=4.6 \AA$ out of plane. The patterns obtained from the $20 \mathrm{~nm}$ thickness film show cubic perovskite-like phase structure with lattice constant of about $a=3.9 \AA$. The diffraction pattern for the [110] zone-axis (Fig. 1b) shows a twofold superstructure, which consists of a perovskite-like cubic phase with vector [100] along [110] direction of the NGO substrate and an unknown phase producing spots with a similar intensity. RHEED patterns of the $60 \mathrm{~nm}$ thickness film demonstrated only the perovskite-like phase and showed no superstructure.

Typical resistivity $(\rho)$ versus temperature $(T)$ data plots of the investigated films exhibit maxima at particular temperatures $\left(T_{\mathrm{m}}\right)$. The value of $T_{\mathrm{m}}$ increases with film thickness up to $20 \mathrm{~nm}$ and then tends to saturate. At low film thickness, 

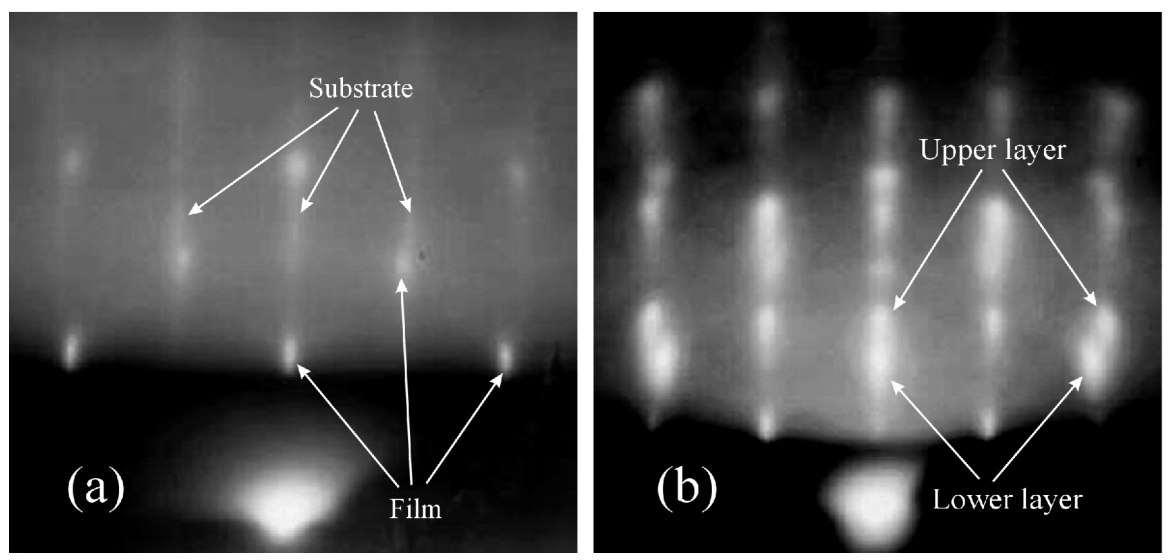

Fig. 1. Electron diffraction patterns recorded from the surface of samples with thicknesses of $4 \mathrm{~nm}$ (a) and $20 \mathrm{~nm}(\mathrm{~b})$.
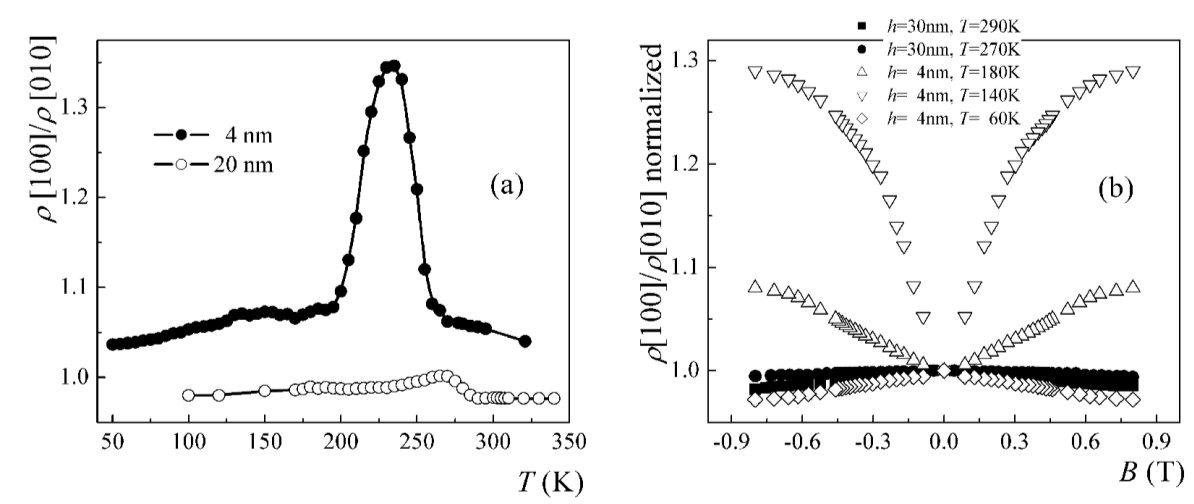

Fig. 2. (a) Temperature dependence of $\rho_{[100]} / \rho_{[010]}$ ratio for two films of different thicknesses. (b) $\rho(B)_{[100]} / \rho(B)_{[010]}$ (normalized to $\rho_{[100]} / \rho_{[010]}$ at zero magnetic field) vs. magnetic field inductance for films with two different thicknesses.

the resistivity is higher in compressed [100] and lower in stressed [010] directions. Figure 2a shows $\rho_{[100]} / \rho_{[010]}$ ratio as a function of $T$ for two films of different thickness. It is evident that anisotropy of the resistivity $(\rho)$ is higher at lower thickness and peaks at $T_{\mathrm{m}}$.

Figure $2 \mathrm{~b}$ shows the ratio $\rho(B)_{[100]} / \rho(B)_{[010]}\left(\right.$ normalized to $\rho_{[100]} / \rho_{[010]}$ ratio obtained at zero magnetic field value) vs. magnetic field inductance $(B)$ for two films with different thickness at various temperatures. MR anisotropy in the $4 \mathrm{~nm}$ thickness film increases when $B$ is increased, reaching its highest value near $T_{\mathrm{m}}$. In contrast, films thicker than $20 \mathrm{~nm}$ demonstrated very little MR anisotropy.

Investigation of ER was performed on films with three critical thicknesses: 8, 20, and $60 \mathrm{~nm}$. For 8 and $60 \mathrm{~nm}$ thick films, the energy of strong electric fields dissipates mainly as a heat, producing a change in the resistance. At temperatures 
below $T_{\mathrm{m}}$, when films exhibit metallic electrical conductivity, the ER effect was positive. However, at $T>T_{\mathrm{m}}$, when the films were in a semiconducting state, the ER was negative. The ER in $20 \mathrm{~nm}$ thick film was negative at all temperatures below $T_{\mathrm{m}}$. Figure 3a,b shows ER (defined as ER $=\{[R(E)-R(0)] / R(0)\} \times$ $100 \%)$ vs. electric field strength $E$ for the $20 \mathrm{~nm}$ film at different temperatures in two perpendicular directions to the substrate plane. Here $R(E)$ and $R(0)$ are resistances of the film in a strong and weak electric field, respectively. The electric field was defined as $E=V / d$, where $V$ is the bias voltage, $d$ is the gap between the contacts.
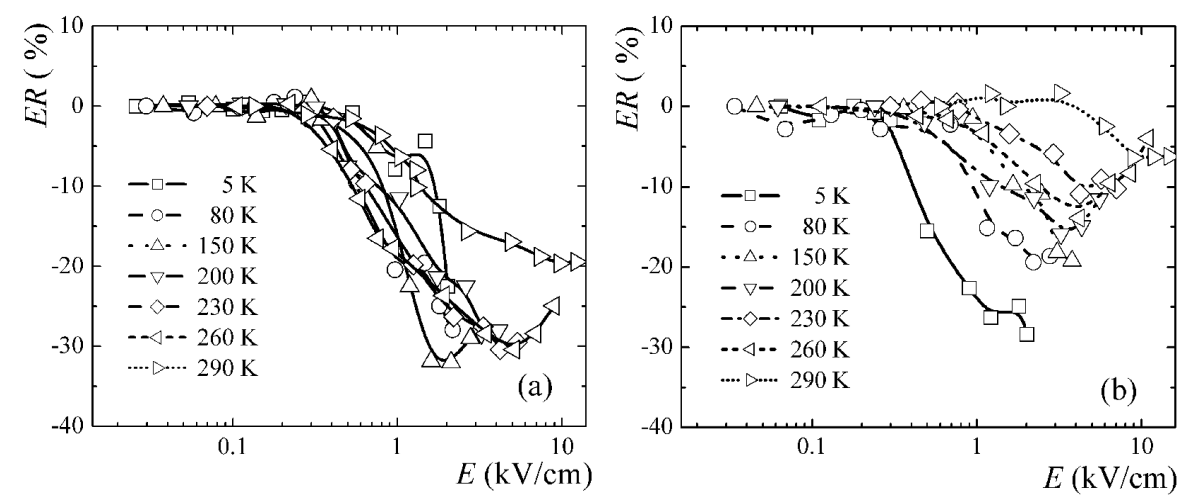

Fig. 3. Electroresistance vs. electric field strength at different temperatures for $20 \mathrm{~nm}$ thickness $\mathrm{La}_{0.83} \mathrm{Sr}_{0.17} \mathrm{MnO}_{3}$ films in two perpendicular directions in the substrate plane, [100] (a) and [010] (b).

In conclusion, the origin of difference in parameters (electrical conductivity and $T_{\mathrm{m}}$ ) between ultrathin and thin $\mathrm{La}_{0.83} \mathrm{Sr}_{0.17} \mathrm{MnO}_{3}$ films comes from the different structure of these films. The low field resistivity and magnetoresistance effects at the substrate-film interface region are anisotropic due to the anisotropy of the strain induced by the $\mathrm{NdGaO}_{3}$ substrate. Films with moderate thickness $(20 \mathrm{~nm})$ exhibit negative electroresistance in strong electric fields due to structural imperfections located between the "dead layer" and the rest of the film.

\section{Acknowledgments}

The work was supported by the Lithuanian Science and Studies Foundation contr. No. K-058.

\section{References}

[1] Y. Lu, X.W. Li, G.Q. Gong, G. Xiao, A. Gupta, Ph. Lecoeur, J.-Z. Sun, Y. Ywang, V.P. Dravid, Phys. Rev. B 54, R8357 (1996).

[2] A.M. Haghiri-Gosnet, J.P. Renard, J. Phys. D, Appl. Phys. 36, R127 (2003).

[3] H.L. Ju, K.M. Krishnan, D. Lederman, J. Appl. Phys. 83, 7073 (1998). 